\title{
EXTENSION OF AN OPPENHEIM TYPE DETERMINANTAL INEQUALITY FOR THE BLOCK HADAMARD PRODUCT
}

\author{
SHENG DONG AND QIAN LI
}

Abstract. Günther and Klotz [Linear Algebra Appl. 437 (2012) 948-956] conjectured an Oppenheim type determinantal inequality for the block Hadamard product of two block commuting positive semidefinite matrices, which is proved by Lin [Linear Algebra Appl. 452 (2014) 1-6]. In this note, we extend the inequality to more block commuting positive semidefinite matrices. And the extension of this type inequality for the Hadamard product is also derived.

Mathematics subject classification (2010): 15A45, 47A63.

Keywords and phrases: Oppenheim type determinantal inequality, the block Hadamard product, the Hadamard product, positive semidefinite matrices.

\section{REFERENCES}

[1] X. Fu AND Y. LiU, Some determinantal inequalities for Hadamard and Fan products of matrices, Journal of Inequalities and Applications, 1, 2016, 262.

[2] M. GÜNTHER AND L. KLOTZ, Schur's theorem for a block Hadamard product, Linear Algebra Appl. 437, 3, 2012, 948-956.

[3] R. A. Horn And C. R. Johnson, Matrix Analysis, 2nd ed., Cambridge University Press, Cambridge.

[4] R. A. Horn, R. Mathias And Y. NakAmura, Inequalities for unitarily invariant norms and bilinear matrix products, Linear Multilinear Algebra, 30, 4, 1989, 303-314.

[5] M. Lin, An Oppenheim type inequalities for a block Hadamard product, Linear Algebra Appl., 452, 7, 2014, 1-6.

[6] G. P. H. STYAN, Hadamard products and multivariate statistical analysis, Linear Algebra Appl., 6, 1973, 217-240. 